\title{
Prognostic significance of XIAP and NF-KB expression in esophageal carcinoma with postoperative radiotherapy
}

\author{
Suna Zhou', Wenguang Ye ${ }^{2}$, Qiuju Shao ${ }^{1}$, Yuhong Qi ${ }^{1}$, Mingxin Zhang ${ }^{2^{*}}$ and Jun Liang ${ }^{1 *}$
}

\begin{abstract}
Background: X-chromosome-linked IAP (XIAP) and nuclear factor-kB (NF-KB) are frequently overexpressed and correlate closely with chemoradiotherapy resistance and poor prognosis in many cancers. However, the significance of XIAP and $\mathrm{NF}-\mathrm{kB}$ expression in radiotherapy sensitivity and its effect on the prognosis of esophageal squamous cell carcinoma (ESCC) are still unknown. The aim of this study was to examine XIAP and NF-KB status in ESCC patients undergoing postoperative radiotherapy after radical surgery, and to evaluate their clinical significance.

Methods: A total of 78 ESCC patients treated with postoperative radiotherapy after radical surgery were enrolled in this study. We immunohistochemically investigated the expression of XIAP and NF-KB in tissues from enrolled patients with specific antibodies. Then, the correlations among XIAP, NF-kB expression, clinicopathological features and its prognostic relevance in ESCC were analyzed.

Results: The increased expression of XIAP and NF-KB in ESCC tissues were clearly correlated with the tumor differentiation and $\mathrm{p}$-TNM stage. Significant positive correlations were found between the expression status of $\mathrm{XIAP}$ and NF-KB $(r=0.779, P=0.000)$. Overexpression of XIAP and NF-KB and metastasis were significantly associated with shorter overall survival times in univariate analysis $(P<0.05)$. Multivariate analysis also confirmed that XIAP expression was an independent prognostic factor $(P=0.005)$.
\end{abstract}

Conclusions: XIAP and NF-KB are intensively expressed in ESCC. The level of XIAP is positively correlated to progression and prognosis of ESCC.

Keywords: Esophageal squamous cell carcinoma, X-chromosome-linked IAP, Nuclear factor-kB

\section{Background}

Esophageal cancer is one of the most aggressive and lethal malignancies, and esophageal squamous cell carcinoma (ESCC) is the major histologic form of esophageal cancer [1]. Surgery is still the mainstay treatment for patients with esophageal cancer, however, the five-year risk of the operable recurrence is $70 \%$ to $80 \%[2,3]$. Postoperative radiotherapy as an adjuvant therapy is being used more often to improve the outcome of ESCC patients after surgery [2]. However, not all patients with ESCC benefit from radiotherapy, and there are individual differences in response to postoperative radiotherapy. The

\footnotetext{
*Correspondence: zm×3115@163.com; Liangjtangdu@163.com

${ }^{2}$ Department of Gastroenterology, Tangdu Hospital, Fourth Military Medical University, Xinsi Road 1, Xi'an, Shaanxi, China

'Department of Radiotherapy, Tangdu Hospital, Fourth Military Medical University, Xinsi Road 1, Xi'an, Shaanxi, China
}

outcomes vary greatly and unpredictably, and the survival of responders has been reported to be better than that of non-responders [4,5]. It is important to recognize the probable treatment response in these tumors. The main obstacle to this approach is the lack of availability of prognostic biomarkers. Pretreatment clinical parameters such as gender, age, TNM classification, and tumor differentiation are not effective in predicting the biologic behavior of ESCC patients who receive postoperative radiotherapy. Thus, it is necessary to identify available biomarkers for predicting response and treatment outcomes of postoperative radiotherapy in ESCC patients.

Among the molecular pathways potentially involved in generating the differential response to radiotherapy, an association has consistently been observed between the apoptotic pathway and the tumor radiosensitivity $[4,5]$. 
Inhibitor of apoptosis (IAP) proteins are a family of endogenous antiapoptotic proteins [6]. Among the eight human IAP proteins, X-chromosome-linked IAP (XIAP) has been reported to exert the most pronounced antiapoptotic function, which has been linked to its ability to bind to caspase-3, -7 and -9 [7]. Firstly, a number of studies have demonstrated that elevated expression levels of XIAP in many types of tumors correlates with a poor prognosis [8]. Secondly, both in vitro and in vivo studies have further demonstrated that downregulation of XIAP expression, either by RNA interference (RNAi) or antisense oligonucleotides, results in stimulation of sensitization to gamma-irradiation and chemotherapeutic-induced apoptosis in tumor cells [9-12]. Likewise, XIAP has been found to be highly expressed in ESCC, and its downregulation by RNAi sensitizes ESCC cell lines to chemotherapeutics [13]. Additionally, more and more research has shown that XIAP acts as a radioresistance factor for radiotherapy in human cancers [14-19]. However, whether XIAP can play a role as a prognostic marker for radiotherapy in ESCC patients has not been extensively investigated to date.

Moreover, aberrant nuclear factor- $\mathrm{k} B(\mathrm{NF}-\mathrm{kB})$ expression has been detected in many human malignancies. $\mathrm{NF}-\mathrm{KB}$ is a transcription factor that regulates the expression of genes linked to inflammation, apoptosis, survival, proliferation, invasion, angiogenesis, metastasis, chemoresistance, tumor cell transformation, and radioresistance [20]. NF- $\mathrm{kB}$ may activate the expression of several genes or proteins that are involved in the apoptotic regulation, such as IAPs [21]. Furthermore, XIAP has also been implicated in the regulation of NF-KB activation [22]. On the other hand, NF-kB may be responsible for blocking the efficacy of chemotherapy and radiation in some types of tumor cells. The positive correlation between NF- $\mathrm{kB}$ expression in ESCC and their resistance to chemoradiation therapy has been previously reported [23], but more specific studies are required to confirm the significance of NF- $\mathrm{kB}$ in predicting disease progression in postoperative radiotherapy of ESCC.

The aim of this study was to determine the prognostic significance of XIAP and NF- $\mathrm{KB}$ in terms of overall survival in ESCC treated with surgery followed by radiotherapy. This was done by using immunohistochemical staining to explore the potential markers in 78 ESCC patients who underwent a surgical resection and postoperative radiotherapy. We also investigated whether the expression levels of XIAP correlate with that of NF- $\mathrm{kB}$ in this patient population.

\section{Methods}

\section{Patients and specimens}

A total of 88 patients with ESCC were selected for this study between January 2000 and December 2007 in the
Tangdu Hospital of Fourth Military Medical University and First Affiliated Hospital of Medical School of Xi'an Jiaotong University. Of these, the tumor staging, clinicopathological information, or follow-up was incomplete for ten patients. As a result, 78 patients were retrospectively reviewed. In addition, patients were required to meet the following criteria: (1) all ESCC cases were pathology confirmed; (2) no distant metastasis (except to the supraclavicular and celiac lymph nodes); (3) the patients completed the whole course of radiotherapy; (4) the patients received preoperative radiotherapy or chemotherapy were excluded; (5) the patients received postoperative chemotherapy or postoperative concurrent chemoradiation were excluded. Tissue samples collected during biopsy and surgery were formalin fixed and paraffin embedded. The Institutional Ethics Committee approval for this study had been obtained from the Tangdu hospital Institutional Review Board.

\section{Surgery}

All patients underwent radical surgery. The surgical approach consisted of a limited thoracotomy on the right side and intrathoracic gastric tube reconstruction (IvorLewis procedure) for lesions in the middle/lower-third of the esophagus. Upper-third lesions were treated by neck anastomosis (Mckeown procedure). Patients underwent two-field or three-field lymph node dissection (the neck, mediastinum and abdomen) depending on the surgical approach used.

\section{Postoperative radiotherapy}

The selection of postoperative adjuvant therapy was made according to the individual physicians' preference and the general physical conditions of the patient. Postoperative radiation was begun three to four weeks after the surgery. All patients were treated using threedimensional conformal radiation therapy (3D-CRT) after esophagectomy. The initial treatment volume included the primary tumor and enlarged lymph nodes. The median radiation dose of 48 Gy (40 to 50 Gy, 2 Gy per fraction, five days per week) was delivered with a three- or four-field technique in 20 to 25 fractions. The extent of the irradiation field was determined based on the primary site in the esophagus. For the lesions of the upper/middle-third of the esophagus, the irradiation area included the tumor bed, bilateral supraclavicular fossae, mediastinum, and subcarinal area; for the lower-third lesions of the esophagus, the tumor bed, bilateral supraclavicular fossae, mediastinum, subcarinal area, and lower thoracic paraesophageal lymph nodes area were irradiated.

\section{Follow-up}

Follow-up evaluations were performed every three to four months after radiotherapy until the study was 
finished or the patient died. The mean duration of follow-up was 23.63 months ( \pm 13.14 months).

\section{Immunohistochemical staining}

Tissue specimens were fixed in neutral buffered formalin $(10 \% \mathrm{v} / \mathrm{v}$ formalin in water; $\mathrm{pH} 7.4)$ and embedded in paraffin wax. Serial sections of $4 \mu \mathrm{m}$ thickness were cut and mounted on charged glass slides. The monoclonal antibody against XIAP (1:100; Cell Signaling Technology, Beverly, MA, USA) and NF-кB (1:200; Santa Cruz Biotechnology, Santa Cruz, CA, USA) were used respectively. The streptavidin-peroxidase technique (Golden Bridge International: SP-9000, Mukilteo, Washington, USA) was used according the manufacturer's instruction. An irrelevant rabbit antiserum served as a negative control. Sections were counterstained with Mayer's hematoxylin.

\section{Immunohistochemical analysis}

Two observers who were blinded to clinical and followup data evaluated staining results independently and coobserved for a consensus when they were divergent. Both the percentage of positive cells and the strength of the staining were considered in the following method. Five degree magnification visions were chosen randomly under the optical microscope, the calculation of results being as followed: the percentage of positive cells in $0 \%$ to $5 \%$ was counted 0 ; the percentage of positive cells in $5 \%$ to $25 \%$ was counted $1 ; 26 \%$ to $50 \%$ was counted 2 ; $51 \%$ to $75 \%$ was counted $3 ; \geq 76 \%$ was counted 4 . In respect of staining strength, the score for tumor cells without stain is 0 ; straw yellow for 1 ; brown for 2; tan for 3 . The staining index score was the sum of the items above. For the purpose of statistical analysis, 3 was used as a cutoff value to distinguish tumors with a low $(<3)$ or high $(\geq 3)$ level of expression.

\section{Statistical analysis}

Data were analyzed using SPSS version 13.0 (SPSS Institute, Chicago, IL, USA). Fisher's exact test was used to analyze the correlation between staining index and other categorical factors potentially predictive of prognosis. The Spearman's rank correlation coefficient was used for analyzing the association of NF- $\mathrm{BB}$ expression levels with XIAP expression status. Overall survival was determined as the time (in months) from the date of surgery to last follow-up or to 1 January 2013, for living patients or to the date of death. Survival curve and median survival were estimated by the Kaplan-Meier method, and the statistical differences between survival curves were examined by the log-rank test. A Cox proportional hazard regression model was used to perform multivariate analyses. $P<0.05$ was regarded as statistically significant.

\section{Results}

Expression of XIAP and NF-KB in ESCC and their relationships to clinicopathological variables

Levels of XIAP and NF- $\mathrm{KB}$ were evaluated by immunohistochemical analysis. XIAP immunoreactivity showed

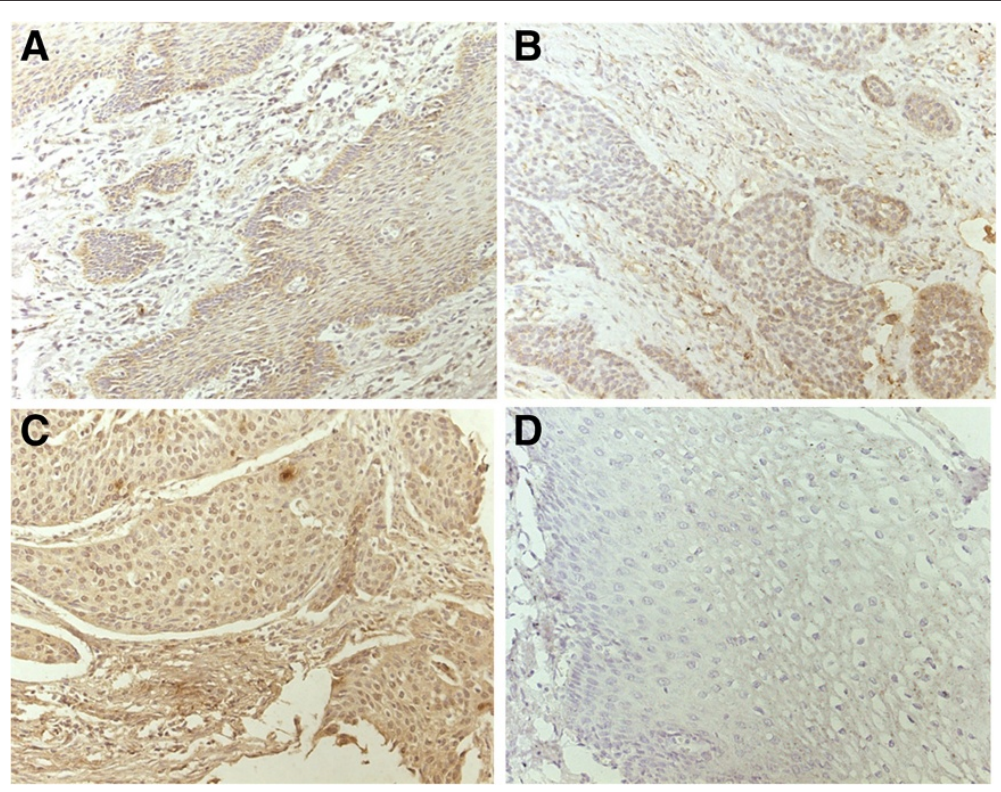

Figure 1 Immunohistochemical expression of X-linked-chromosome inhibitor of apoptosis (XIAP) protein and nuclear factor-KB (NF-KB) in esophageal squamous cell carcinoma (ESCC). (A) typical immunohistological features with high levels of XIAP expression in ESCC. The XIAP staining shown cytoplasmic localization; (B) typical immunohistological features with lower levels of XIAP expression in ESCC; (C) typical immunohistological features with high levels of NF-KB in ESCC. The NF-KB staining was present in the nucleus and cytoplasm of tumor cells; (D) negative staining in ESCC. Magnifications $\times 200$. 
cytoplasmic localization, while NF- $\mathrm{kB}$ was mainly located in the nucleus and cytoplasm. Figure 1 shows representative expression patterns of XIAP and NF- $\mathrm{kB}$ in ESCC. The increased expression of XIAP and NF- $\mathrm{KB}$ in ESCC tissues showed obvious correlation with the tumor differentiation and p-TNM stage (Table 1).

\section{Association of XIAP expression levels with NF-KB expression status}

Since XIAP can activate the transcription factor NF$\kappa \mathrm{B}$, a known survival factor for cancer cells, we next investigated the association of XIAP expression levels with NF- $\mathrm{B}$ expression status (Table 2). Of the $54 \mathrm{tu}-$ mors containing a high level of cytoplasmic XIAP immunoreactivity, a total of 51 cases displayed a high

Table 1 Correlation of X-linked-chromosome inhibitor of apoptosis (XIAP) protein and nuclear factor-KB (NF-KB) expression to clinicopathologic characteristics of 78 patients with esophageal squamous cell carcinoma (ESCC)

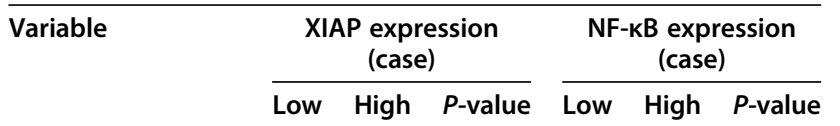

Gender

Male

Female

Age

$<60$

$\geq 60$

Low High $P$-value

Low High $P$-value

ale

$\begin{array}{ll}15 & 33 \\ 8 & 22\end{array}$

0.800

$12 \quad 36$

1.000

Tumor location

Lower thoracic

Middle and Upper

thoracic

Length of tumor

\begin{tabular}{lcccccc}
$<5 \mathrm{~cm}$ & 14 & 30 & 0.628 & 10 & 34 & 0.603 \\
$\geq 5 \mathrm{~cm}$ & 9 & 25 & & 10 & 24 & \\
$\begin{array}{l}\text { T-stage } \\
\mathrm{T}_{3}\end{array}$ & 12 & 41 & 0.066 & 14 & 39 & 1.000 \\
$\mathrm{~T}_{1-2}$ & 11 & 14 & & 6 & 19 & \\
$\mathrm{~N}$-stage & & & & & & \\
$\mathrm{N}_{0}$ & 11 & 22 & 0.618 & 11 & 22 & 0.201 \\
$\mathrm{~N}_{1}$ & 12 & 33 & & 9 & 36 & \\
P-TNM stage & & & & & & \\
I-II & 13 & 15 & 0.020 & 12 & 16 & 0.014 \\
III & 10 & 40 & & 8 & 42 & \\
Differentiation & & & & & & \\
Well and Medium & 23 & 28 & 0.000 & 20 & 31 & 0.000 \\
Poor & 0 & 27 & & 0 & 27 & \\
\hline
\end{tabular}

Table 2 Association of nuclear factor-KB (NF-KB) expression levels with $\mathrm{X}$-linked-chromosome inhibitor of apoptosis (XIAP) protein expression status

\begin{tabular}{|c|c|c|c|c|c|}
\hline \multirow[t]{2}{*}{ Variables } & \multirow[t]{2}{*}{ Total } & \multicolumn{2}{|l|}{ XIAP } & \multirow[t]{2}{*}{$P$} & \multirow[t]{2}{*}{$r$} \\
\hline & & Low & High & & \\
\hline NF-KB & & & & 0.000 & 0.779 \\
\hline Low & 20 & 18 & 2 & & \\
\hline High & 58 & 5 & 53 & & \\
\hline
\end{tabular}

expression of NF-кB. We calculated the Spearman's rank correlation coefficient to evaluate the linear relationship. There was statistically significant association of XIAP expression status with NF- $\mathrm{kB}$ expression levels $(\mathrm{r}=0.779, P=0.000)$.

Table 3 Univariate analysis for overall survival

\begin{tabular}{|c|c|c|c|c|}
\hline \multirow[t]{2}{*}{ Variables } & \multirow[t]{2}{*}{ Total } & \multicolumn{2}{|c|}{ Overall survival } & \multirow[t]{2}{*}{$P$} \\
\hline & & Median \pm SE & $95 \% \mathrm{Cl}$ & \\
\hline XIAP & & & & 0.000 \\
\hline Low & 23 & $44.00 \pm 0.99$ & 42.05 to 45.95 & \\
\hline High & 55 & $20.00 \pm 1.30$ & 17.45 to 22.55 & \\
\hline $\mathrm{NF}-\mathrm{KB}$ & & & & 0.000 \\
\hline Low & 20 & $44.00 \pm 1.20$ & 41.66 to 46.34 & \\
\hline High & 58 & $20.00 \pm 1.37$ & 17.32 to 22.68 & \\
\hline Gender & & & & 0.624 \\
\hline Male & 48 & $22.00 \pm 1.79$ & 18.48 to 25.52 & \\
\hline Female & 30 & $30.00 \pm 4.71$ & 20.77 to 39.23 & \\
\hline Age & & & & 0.956 \\
\hline$<60$ & 48 & $22.00 \pm 3.10$ & 15.92 to 28.08 & \\
\hline$\geq 60$ & 30 & $26.00 \pm 5.11$ & 15.98 to 36.02 & \\
\hline Tumor location & & & & 0.421 \\
\hline Lower thoracic & 44 & $22.00 \pm 7.37$ & 7.56 to 36.44 & \\
\hline Middle and Upper thoracic & 34 & $40.13 \pm 5.68$ & 19.58 to 28.42 & \\
\hline Length of tumor & & & & 0.421 \\
\hline$<5 \mathrm{~cm}$ & 44 & $24.00 \pm 2.25$ & 19.58 to 28.42 & \\
\hline$\geq 5 \mathrm{~cm}$ & 34 & $22.00 \pm 7.37$ & 7.56 to 36.44 & \\
\hline T-stage & & & & 0.202 \\
\hline $\mathrm{T}_{3}$ & 53 & $24.00 \pm 2.59$ & 18.93 to 29.07 & \\
\hline$T_{1-2}$ & 25 & $29.00 \pm 5.34$ & 18.53 to 39.47 & \\
\hline $\mathrm{N}$-stage & & & & 0.238 \\
\hline $\mathrm{N}_{0}$ & 33 & $24.00 \pm 7.65$ & 9.01 to 38.99 & \\
\hline $\mathrm{N}_{1}$ & 45 & $24.00 \pm 2.93$ & 18.25 to 29.75 & \\
\hline p-TNM stage & & & & 0.076 \\
\hline$|-| \mid$ & 28 & $32.00 \pm 2.82$ & 26.48 to 37.52 & \\
\hline III & 50 & $20.00 \pm 2.18$ & 15.74 to 24.26 & \\
\hline Differentiation & & & & 0.000 \\
\hline Well and Medium & 51 & $34.00 \pm 3.40$ & 27.35 to 40.66 & \\
\hline Poor & 27 & $16.00 \pm 2.43$ & 11.24 to 20.76 & \\
\hline
\end{tabular}



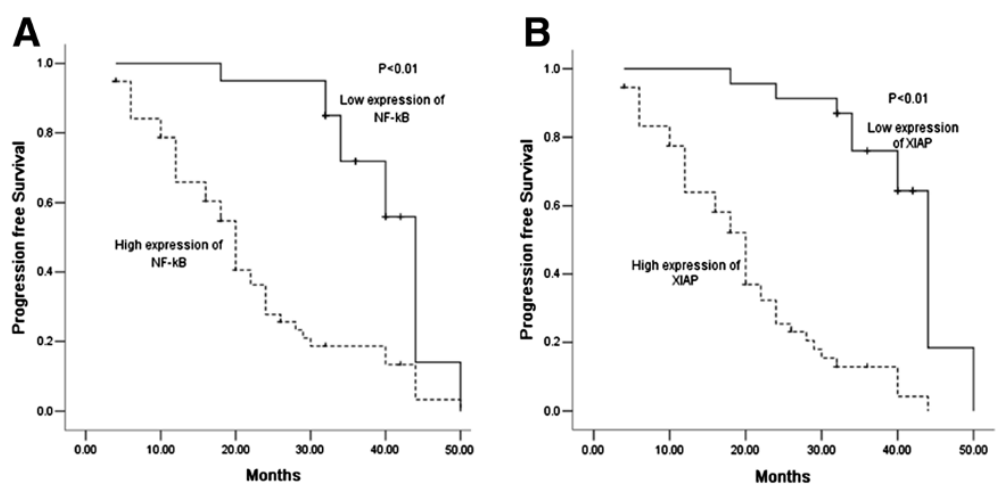

Figure 2 Kaplan-Meier survival analysis. Progression free survival differences between patients with high and low levels of protein expression or metastasis. P-value was obtained using the log-rank test of the difference. (A) X-linked-chromosome inhibitor of apoptosis (XIAP) protein; (B) Nuclear factor- $\mathrm{kB}(\mathrm{NF}-\mathrm{kB})$.

\section{Survival analysis}

Kaplan-Meier analysis was used to calculate the impact of classic clinicopathologic features and protein expression on survival (Table 3, Figure 2). Differentiation and high expression of XIAP and NF-kB were associated with decreased survival $(P<0.05)$, whereas other clinicopathological variables were not significant. Cox regression analysis revealed a statistically significant correlation among differentiation and XIAP expression $(P<0.05$, Table 4).

\section{Discussion}

Compared with the surgery alone, earlier studies have suggested that postoperative prophylactic radiotherapy produces a better prognosis in esophageal cancer patients [24-26]. However, not all patients benefit from radiotherapy; some even have a shorter survival time. A good tumor response is usually responsible for an excellent prognosis. Thus, biomarkers that can predict radiation response and outcomes before treatment are needed and a high level of apoptotic tumor cells is a predictive marker for tumor response in antitumor therapy. Among the numerous proteins involved in apoptosis regulation, IAP proteins play an important role. The human IAP family is composed of eight proteins: NAIP (BIRC1), c-IAP1 (BIRC2), c-IAP2 (BIRC3), XIAP (BIRC4), survivin (BIRC5), Apollon/Bruce (BIRC6), ML-IAP (BIRC7 or livin) and ILP-2 (BIRC8). Furthermore, XIAP is the only member of this family able to directly inhibit both the initiation and execution phase of the caspase cascade

Table 4 Multivariate Cox proportional hazards analysis for overall survival

\begin{tabular}{cccc}
\hline Variables & \multicolumn{2}{c}{ Overall survival } & $\boldsymbol{P}$ \\
\cline { 2 - 3 } & $\mathbf{R R}$ & $\mathbf{9 5 \%} \mathbf{~ C l}$ & \\
\hline XIAP & 4.68 & 1.60 to 13.67 & 0.005 \\
Differentiation & 3.55 & 1.51 to 8.37 & 0.004 \\
\hline
\end{tabular}

which is crucial to mediate the controlled demise of malignant cells. More widely, XIAP has been found to be frequently overexpressed in most human cancer cells and renders cells resistant to cancer treatment and so XIAP has received interest as a therapeutic target $[9,27]$. Recently, increasingly more research studies have found that small-molecule XIAP inhibitors could enhance irradiation-induced apoptosis in most cancer cells [28-30]. However, the prognosis of XIAP for postoperative prophylactic radiotherapy in ESCC patients has not been extensively investigated. On the other hand, the most important contribution of IAPs to cell survival and tumorigenesis resides in the ability of XIAP to regulate ubiquitin-dependent activation of NF- $\kappa B$ [31]. The high constitutive nuclear activation of NF- $\mathrm{kB}$ activation has been detected in many solid cancers, which attribute mainly to the development and progression of cancer such as proliferation, migration and apoptosis [21]. A positive correlation was observed between NF- $\mathrm{kB}$ and nodal metastasis in ESCC, but the clinical significance for NF- $\mathrm{kB}$ in predicting associated outcomes and its correlation with XIAP in patients receiving postoperative radiotherapy for ESCC have not been reported before.

In this study, we found that XIAP and NF- $\mathrm{kB}$ were highly expressed in ESCC specimens. Immunostaining showed the expression of XIAP in the resected ESCC specimens to be mostly in the cytoplasm, whereas NF$\mathrm{\kappa B}$ expression was predominantly localized in the nucleus (Figure 1). Consistently, our results showed that the high level of XIAP expression correlated significantly with both tumor differentiation and p-TNM stage, and the same results were obtained for NF-kB. Next, the statistical analysis shows that the in vivo correlation between XIAP and NF- $\kappa \mathrm{B}$ expression is a highly significant association. This means NF- $\mathrm{kB}$ may be one of XIAP-related interacting partners. However, the exact molecular basis by which XIAP might interact with $\mathrm{NF}-\kappa \mathrm{B}$ remains to be clarified in vivo. Overall, the 
available data so far suggest that XIAP pathway may well also be related to the genetic changes implicated in ESCC radiosensitivity.

Previous investigation has largely focused on the prognostic value of NF- $k B$ in solid cancer, but there have been few reports about that of XIAP [19,32,33]. Moreover, the prognostic value of XIAP for radiotherapy in ESCC is not clear. Based on our observation of 78 patients with ESCC, our study explores the hypothesis that overexpression of XIAP and NF- $\mathrm{KB}$ is predictive of shorter survival in ESCC patients following surgery and postoperative. It is generally considered that patients will benefit greatly from antitumor therapy if available accurate information about the likely outcomes before treatment is started can be obtained. Consequently, further study is needed to elucidate markers or combinations of markers which best reflect the effects of radiotherapy in ESCC. Such markers might prove valuable not only as clinical predictors, but also as targets for ESCC treatment: for example, treatment might result in increased sensitivity if these abnormalities of function and expression return to normal.

\section{Conclusion}

In conclusion, high level of XIAP expression was an independent unfavorable prognostic indicator in ESCC patients treated with radiotherapy after surgery. This might be useful in helping clinicians to choose, with more accuracy, the best clinical policy and therapy in patients. Furthermore, we will be able to focus in future on both the prognostic and treatment value of XIAP.

\section{Abbreviations}

XIAP: X-chromosome-linked IAP; NF-KB: Nuclear factor-KB; ESCC: Esophageal squamous cell carcinoma; RNAi: RNA interference; 3D-CRT: Three-dimensional conformal radiation therapy; RR: Relative risk.

\section{Competing interests}

The authors declare that they have no competing interests.

\section{Authors' contributions}

SZ analyzed the data and wrote the manuscript. MZ and JL commented on and revised the manuscript. WY, QS and YQ built the patient database. All authors read and approved the final manuscript.

\section{Acknowledgments}

This work was supported by Chinese National Natural Science Foundation Projects (NSFC 81301922 and 81302055).

Received: 25 May 2013 Accepted: 25 October 2013

Published: 5 November 2013

\section{References}

1. Hiyama T, Yoshihara M, Tanaka S, Chayama K: Genetic polymorphisms and esophageal cancer risk. Int J Cancer 2007, 121(8):1643-1658.

2. Yuequan J, Shifeng C, Bing Z: Prognostic factors and family history for survival of esophageal squamous cell carcinoma patients after surgery. Ann Thorac Surg 2010, 90(3):908-913.

3. Nakagawa S, Kanda T, Kosugi S, Ohashi M, Suzuki T, Hatakeyama K Recurrence pattern of squamous cell carcinoma of the thoracic esophagus after extended radical esophagectomy with three-field lymphadenectomy. J Am Coll Surg 2004, 198(2):205-211.

4. Miyazaki T, Kato H, Faried A, Sohda M, Nakajima M, Fukai Y, Masuda N, Manda R, Fukuchi M, Ojima H, Tsukada K, Kuwano H: Predictors of response to chemo-radiotherapy and radiotherapy for esophageal squamous cell carcinoma. Anticancer Res 2005, 25(4):2749-2755.

5. Li BZ, Chen ZL, Shi SS, Feng XL, Tan XG, Zhou F, He J: Overexpression of $\mathrm{Cdc} 25 \mathrm{C}$ predicts response to radiotherapy and survival in esophageal squamous cell carcinoma patients treated with radiotherapy followed by surgery. Chin J Cancer 2013. doi:10.5732/cjc.012.10233. [Epub ahead of print].

6. Fulda S, Vucic D: Targeting IAP proteins for therapeutic intervention in cancer. Nat Rev Drug Discov 2012, 11(2):109-124.

7. Eckelman BP, Salvesen GS, Scott FL: Human inhibitor of apoptosis proteins: why XIAP is the black sheep of the family. EMBO Rep 2006, 7(10):988-994

8. Vucic D: Targeting IAP, (inhibitor of apoptosis) proteins for therapeutic intervention in tumors. Curr Cancer Drug Targets 2008, 8(2):110-117.

9. Sasaki H, Sheng Y, Kotsuji F, Tsang BK: Down-regulation of X-linked inhibitor of apoptosis protein induces apoptosis in chemoresistant human ovarian cancer cells. Cancer Res 2000, 60(20):5659-5666.

10. Hu Y, Cherton-Horvat G, Dragowska V, Baird S, Korneluk RG, Durkin JP, Mayer LD, LaCasse EC: Antisense oligonucleotides targeting XIAP induce apoptosis and enhance chemotherapeutic activity against human lung cancer cells in vitro and in vivo. Clin Cancer Res 2003, 9(7):2826-2836.

11. Bilim V, Kasahara T, Hara N, Takahashi K, Tomita Y: Role of XIAP in the malignant phenotype of transitional cell cancer (TCC) and therapeutic activity of XIAP antisense oligonucleotides against multidrug-resistant TCC in vitro. Int I Cancer 2003, 103(1):29-37.

12. McManus DC, Lefebvre CA, Cherton-Horvat G, St-Jean M, Kandimalla ER, Agrawal S, Morris SJ, Durkin JP, Lacasse EC: Loss of XIAP protein expression by RNAi and antisense approaches sensitizes cancer cells to functionally diverse chemotherapeutics. Oncogene 2004, 23(49):8105-8117.

13. Zhang S, Ding F, Luo A, Chen A, Yu Z, Ren S, Liu Z, Zhang L: XIAP is highly expressed in esophageal cancer and its downregulation by RNAi sensitizes esophageal carcinoma cell lines to chemotherapeutics. Cancer Biol Ther 2007, 6(6):973-980.

14. Ohnishi K, Scuric Z, Schiestl RH, Okamoto N, Takahashi A, Ohnishi T: siRNA targeting NBS1 or XIAP increases radiation sensitivity of human cancer cells independent of TP53 status. Radiat Res 2006, 166(3):454-462.

15. Kim DW, Seo SW, Cho SK, Chang SS, Lee HW, Lee SE, Block JA, Hei TK, Lee FY: Targeting of cell survival genes using small interfering RNAs (siRNAs) enhances radiosensitivity of Grade II chondrosarcoma cells. J Orthop Res 2007, 25(6):820-828.

16. Giagkousiklidis S, Vellanki SH, Debatin KM, Fulda S: Sensitization of pancreatic carcinoma cells for gamma-irradiation-induced apoptosis by XIAP inhibition. Oncogene 2007, 26(49):7006-7016.

17. Wang R, Li B, Wang X, Lin F, Gao P, Cheng SY, Zhang HZ: Inhibiting XIAP expression by RNAi to inhibit proliferation and enhance radiosensitivity in laryngeal cancer cell line. Auris Nasus Larynx 2009, 36(3):332-339.

18. Connolly K, Mitter R, Muir M, Jodrell D, Guichard S: Stable XIAP knockdown clones of HCT116 colon cancer cells are more sensitive to TRAIL, taxanes and irradiation in vitro. Cancer Chemother Pharmacol 2009, 64(2):307-316.

19. Moussata D, Amara S, Siddeek B, Decaussin M, Hehlgans S, Paul-Bellon R, Mornex F, Gerard JP, Romestaing P, Rödel F, Flourie B, Benahmed M, Mauduit C: XIAP as a radioresistance factor and prognostic marker for radiotherapy in human rectal adenocarcinoma. Am J Pathol 2012, 181(4):1271-1278.

20. Ahn KS, Aggarwal BB: Transcription factor NF-kappaB: a sensor for smoke and stress signals. Ann N Y Acad Sci 2005, 1056:218-233.

21. Dolcet X, Llobet D, Pallares J, Matias-Guiu X: NF-kB in development and progression of human cancer. Virchows Arch 2005, 446(5):475-482.

22. Lu M, Lin SC, Huang Y, Kang YJ, Rich R, Lo YC, Myszka D, Han J, Wu H: XIAP induces NF-kappaB activation via the BIR1/TAB1 interaction and BIR1 dimerization. Mol Cell 2007, 26(5):689-702.

23. Hatata T, Higaki K, Tatebe S, Shomori K, Ikeguchi M: Immunohistochemical study of nuclear factor-KB expression in esophageal squamous cell carcinoma: prognostic significance and sensitivity to treatment with 5FU. Dis Esophagus 2012, 25(8):716-722.

24. Xiao ZF, Yang ZY, Liang J, Miao YJ, Wang M, Yin WB, Gu XZ, Zhang DC, Zhang $R G$, Wang $L$ : Value of radiotherapy after radical surgery for 
esophageal carcinoma: a report of 495 patients. Ann Thorac Surg 2003, 75(2):331-336.

25. Chen J, Zhu J, Pan J, Zhu K, Zheng X, Chen M, Wang J, Liao Z: Postoperative radiotherapy improved survival of poor prognostic squamous cell carcinoma esophagus. Ann Thorac Surg 2010, 90(2):435-442.

26. Chen J, Pan J, Zheng X, Zhu K, Li J, Chen M, Wang J, Liao Z: Number and location of positive nodes, postoperative radiotherapy, and survival after esophagectomy with three-field lymph node dissection for thoracic esophageal squamous cell carcinoma. Int J Radiat Oncol Biol Phys 2012, 82(1):475-482

27. Vogler M, Walczak H, Stadel D, Haas TL, Genze F, Jovanovic M, Bhanot U, Hasel C, Möller P, Gschwend JE, Simmet T, Debatin KM, Fulda S: Small molecule XIAP inhibitors enhance TRAIL-induced apoptosis and antitumor activity in preclinical models of pancreatic carcinoma. Cancer Res 2009, 69(6):2425-2434.

28. Cao C, Mu Y, Hallahan DE, Lu B: XIAP and survivin as therapeutic targets for radiation sensitization in preclinical models of lung cancer. Oncogene 2004, 23(42):7047-7052.

29. Vellanki SH, Grabrucker A, Liebau S, Proepper C, Eramo A, Braun V, Boeckers T, Debatin KM, Fulda S: Small-molecule XIAP inhibitors enhance gammairradiation-induced apoptosis in glioblastoma. Neoplasia 2009, 11(8):743-752.

30. Ohnishi K, Nagata Y, Takahashi A, Taniguchi S, Ohnishi T: Effective enhancement of $X$-ray-induced apoptosis in human cancer cells with mutated p53 by siRNA targeting XIAP. Oncol Rep 2008, 20(1):57-61.

31. Gyrd-Hansen M, Meier P: IAPs: from caspase inhibitors to modulators of NF-kappaB, inflammation and cancer. Nat Rev Cancer 2010, 10(8):561-574.

32. Ramp U, Krieg T, Caliskan E, Mahotka C, Ebert T, Willers R, Gabbert HE, Gerharz CD: XIAP expression is an independent prognostic marker in clear-cell renal carcinomas. Hum Pathol 2004, 35(8):1022-1028.

33. Li M, Song T, Yin ZF, Na YQ: XIAP as a prognostic marker of early recurrence of nonmuscular invasive bladder cancer. Chin Med I (Engl) 2007, 120(6):469-473.

doi:10.1186/1477-7819-11-288

Cite this article as: Zhou et al.: Prognostic significance of XIAP and NF-KB expression in esophageal carcinoma with postoperative radiotherapy. World Journal of Surgical Oncology 2013 11:288.

\section{Submit your next manuscript to BioMed Central and take full advantage of:}

- Convenient online submission

- Thorough peer review

- No space constraints or color figure charges

- Immediate publication on acceptance

- Inclusion in PubMed, CAS, Scopus and Google Scholar

- Research which is freely available for redistribution 